\title{
Contenidos y procesos en pedagogía social: deconstrucción de la última etapa de producción
}

\section{Contents and processes in Social Pedagogy: the deconstruction of the latest research in the field}

\author{
José Antonio Rabadán Rubio ${ }^{1}$ \\ jrabadan@um.es \\ Encarnación Hernández Pérez \\ encarnacion.hernandez2@um.es \\ Universidad de Murcia, España
}

\begin{abstract}
:
When addressing Social Pedagogy contents and processes it is necessary to highlight that this is not a trivial or simple matter, since it is knowledge subject to constant re-elaboration. In most cases, practice is far ahead of theoretical reflection and action should have even more weight than the theory itself. Despite these difficulties, our aim in this article is to analyze the scientific production by scholarly research in the field of Social Education in our country looking at its most relevant vias of expression, The Journal of Social Pedagogy and The Inter-University Seminar on Social Pedagogy. To do this, we will review existing research, contents and processes for the purpose of mapping out the latest contributions to the discipline. We aim to provide objective and scientific knowledge on the development
\end{abstract}

1 Dirección para correspondencia (Correspondence address):

José Antonio Rabadán Rubio. Dpto. de Teoría e Historia de la Educación. Facultad de Educación. Universidad de Murcia. Campus de Espinardo. 30100 Murcia (España). 
Contenidos y procesos en pedagogía social: deconstrucción de la última etapa de producción

José Antonio Rabadán Rubio y Encarnación Hernández Pérez

la última etapa en la producción científica de esta disciplina. Con ello, pretendemos proporcionar un conocimiento objetivo y científico sobre el desarrollo de esta ciencia pedagógica, su realidad actual y sus nuevos retos ante las dinámicas sociales imperantes.

\section{Palabras clave:}

Pedagogía Social; orientación investigadora; dinámicas sociales; producción científica. of this teaching science, its current reality, and its new challenges in light of prevailing social dynamics.

\section{Key words:}

Social Pedagogy; researcher character; social dynamics; scientific output.

\section{Résumé:}

Quand on aborde le contenu et le processus de pédagogie sociale, il est clair qu'il ne $s^{\prime}$ agit pas d'une mince ou simple affaire puisqu'il s'agit $d^{\prime} u n$ savoir en construction permanente. Dans la plupart des cas, la pratique est loin de la réflexion théorique, et I'action devrait même avoir un poids plus important que la théorie elle-même. Malgré les difficultés, notre objectif dans cet article est d'analyser les productions scientifiques des professionnels qui travaillent dans le domaine de l'éducation sociale dans notre pays, à travers ses organes compétents d'expression, Revue internationale de pédagogie sociale Séminaire inter-universitaire sur la pédagogie sociale. Pour ce faire, nous passerons en revue la recherche, le contenu et les processus de la discipline, réalisant ainsi un bref aperçu de la dernière étape de la production scientifique de cette discipline. Avec cela, nous visons à fournir une connaissance objective et scientifique sur le développement de cette science pédagogique, de sa réalité actuelle et de ses nouveaux défis face à la dynamique sociale en vigueur.

\section{Mots clés:}

Pédagogie Sociale; chercheur de caractère; dynamique sociale; production scientifique.

Fecha de recepción: 2-9-2013

Fecha de aceptación: 17-9-2013

\section{Introducción}

Dentro del ámbito de conocimiento de las Ciencias de la Educación, la Educación Social, y, por tanto, la Pedagogía Social, es la disciplina que mayor necesidad de revisión de su fundamentación tiene hoy, debido fundamentalmente a la gran dependencia que tienen ambas con respecto a la propia sociedad. Si entendemos la Pedagogía Social como nuestra conciencia Pedagógica, como nos indicaba Bollnow (1969), es evidente que las importantes trasformaciones sociales que está hoy viviendo nuestro país, nos obligan a una revisión epistemológica, metodológica e, incluso, de usuarios de la propia acción pedagógica, constantes, a la vez que debiera ser tema de controversia y replanteamiento permanente. 
En el desarrollo de estos últimos años, en la Pedagogía Social, hemos vivido una respuesta académica, social y profesional, a la vez que el nacimiento y detección de nuevas necesidades educativas que la sociedad demandaba. En la actualidad parece no ser así. La crisis que venimos atravesando a nivel internacional desde el año 2007, es de una extraordinaria complejidad y sus efectos sobre algunos rasgos básicos de nuestra sociedad no han hecho sino comenzar. Existe el riesgo de que la crisis financiera esté evolucionando hacia una crisis de cohesión social a partir de 2010, al menos en ciertos países como España y, en este proceso de transformación interactúan factores diversos que se retroalimentan mutuamente, provocando así un desplazamiento en las necesidades de la propia sociedad, incluidas las pedagógicas.

Este cambio social nos ha de obligar a realizar un cambio fundamental en nuestro ámbito disciplinar. Hoy ya los actores, los usuarios y recursos disponibles para nuestra "conciencia pedagógica", no son los de antaño. Así, hemos de provocar una transformación de forma inminente dando respuesta a la "verdadera sociedad" cuyas voces hoy están representadas por altísimas cuotas de desempleo, de marginación y de emigración.

La resolución satisfactoria de los actuales problemas científicos y sus derivaciones tecnológicas, económicas y sociales, requiere un enfoque que supere el especialismo y mucho más el esnobismo de algunas especialidades de la propiedad intelectual de parcelas de investigación. La interdisciplinariedad parece ser la única vía practicable en los próximos decenios. Sin embargo, carecemos todavía de elaboraciones epistemológicas adecuadas a la magnitud del problema que enfrenta la práctica científica, lo que determina perplejidades, contradicciones e indecisiones que llegan a ser graves.

Esto nos conduce a concluir, pragmáticamente, que el campo de la ciencia es móvil, y no hablemos en el caso de la Pedagogía Social, y que al menos tenemos que contar con esta realidad - un concepto problemático de "ciencia" - cuando hablemos de "interdisciplinariedad".

\section{Construcción de la disciplina}

A estas alturas de la evolución de los planes de estudio en España de Pedagogía/Ciencia/s de la Educación, es evidente que la Pedagogía Social 
constituye una de las sub-áreas más potentes, del Área de Conocimiento que, formalmente, se denomina Teoría e Historia de la Educación. La comunidad científica, que se encuentra profesionalmente trabajando alrededor de ámbitos relacionados directamente con esta denominación, acepta, de modo más o menos consensuado y a nivel general, que la Pedagogía Social se ha ido conformando como un campo disciplinar que va creciendo y aumentado a medida que se va obteniendo conocimiento sobre el mismo por las diversas vías que poseen los seres humanos. De tal modo que, en este momento, cabría hablar, en ese afán de por hacerse comprender, que uno de los usos de la expresión "Pedagogía Social", además del asociado a una disciplina concreta, es el de hacer referencia a una especie de "matriz disciplinar", comunidad disciplinar o disciplina en leguaje de Becher (2001), una plataforma amplia de conocimientos que genera diferentes líneas de reflexión teóricas orientadas, en gran medida, a la formación de profesionales de la acción educativa. Así, tal y como se puede ver en el temario de Pedagogía Social, esta asumiría una doble traducción:

1. Como campo de conocimiento más o menos amplio, como "el tronco" que da cobertura, o trata de darla, a toda una serie de "ramas" de conocimientos acotadas convencionalmente en disciplinas que se distribuyen, a lo largo de los diversos cursos donde se ubiquen, en el currículo formativo ofertado para cada titulación. De esta misma manera, la Pedagogía Social actuaría como un "paraguas" en sentido global, en el que especificaciones más concretas, otras disciplinas, se articularían en torno a ella. Así, desde esta materia de estudio e investigación, está explicitando un significativo desarrollo teórico, con frecuencia más inarticulado y magmático en unas parcelas que en otras, que precisa de un buen estudio en el que se identifique su capacidad de articulación.

2. Como materia de estudio o disciplina concreta y específica que forma parte de un currículo en el que juega su papel con otras disciplinas, es la de verse convocada a informar y formar a unos alumnos a los que trata de capacitar para que ejerzan como profesionales en las áreas de intervención donde actúan los educadores sociales que se especializan en esta área de conocimiento teórico.

Hasta aquí, la producción científica de la disciplina Pedagogía Social, ha logrado construir un corpus de conocimiento, tanto teórico como profesional, valioso y significativo. Para ello, ha utilizado fundamental- 
mente tres vías de producción. En la primera de ellas, encontramos la promoción de manuales orientados al aprendizaje de los alumnos. Una segunda vía, orientada a una mayor especialización dependiente de los ámbitos de acción y, una tercera, y no por ello menos importante, en la que se ofertan instrumentos, tanto teóricos como prácticos, a los profesionales de la acción educativa-social.

Es evidente que en el devenir de la disciplina, las vías anteriormente citadas corresponderían a las tres vías de aproximación a la construcción de la disciplina, que nos indica Millán Arroyo (1985):

a. Camino Histórico, por el que se trata de bucear en el pasado para estudiar las obras clásicas, y otras no tan fundamentales, de aqueIlos que han contribuido al desarrollo de la Pedagogía Social.

"El planteamiento histórico...nos ofrece la oportunidad de beneficiarnos con la sabiduría de lo pasado. Nos permite entender asuntos que solo podemos captar bien si entendemos sus antecedentes". (Inkeles, 1968)

b. Camino empírico. Es la vía más convocada, aunque la menos seguida. Como apuntaba Millán Arroyo (1985) "es el estudio de la praxis" el que puede alimentar la discusión científica, teórica en Pedagogía Social. Centrando la cuestión que nos planteaba Millán Arroyo, que a la vez sería el punto clave que nos indicaría la dirección de esta vía: ¿Qué es lo que están haciendo hoy los prácticos de la educación social?

c. Camino analítico. Un saber es consistente cuando alcanza a dar razón por sí mismo de su objeto de estudio. El camino analítico seguirá siendo esperanzador a la hora de aproximarnos a la construcción de las disciplinas mientras nos preguntemos "¿Qué es lo que nos sugiere la razón?" ante los diversos hechos que observamos y trate de dar las respuestas más veraces posibles a un argumento siempre abierto: buscar la consistencia científica, sin pretender, por ello, un conocimiento exhaustivo.

Todas las vías citadas, nos permiten sintetizar los contenidos abordados en los diferentes trabajos, artículos y comunicaciones presentadas por los profesionales de la Pedagogía Social, en cuatro ramas de conocimiento: epistemología, sociología, psicología y pedagogía. 
Epistemología. Tipo de conocimiento denominado episteme. Alude al conocimiento reflexivo, elaborado con rigor. De ahí que el término "epistemología" se haya utilizado con frecuencia como equivalente a "ciencia o teoría del conocimiento".

Sociología. Ciencia que estudia los fenómenos colectivos producidos por la actividad social de los seres humanos, dentro del contexto histórico-cultural en el que se encuentran inmersos.

Psicología. Ciencia que estudia la conducta o los comportamientos de los individuos.

Pedagogía. Ciencia que tiene como objeto de estudio a la educación. Ciencia multidisciplinaria, que pertenece al campo de las ciencias sociales y humanidades, tiene por objeto analizar y comprender el fenómeno de la educación, intrínseco a la especie humana, basado en procesos sistemáticos de aprendizaje, conocimiento, desarrollo de capacidades y habilidades, que facilitan la toma de decisiones.

Estas cuatro ramas de conocimiento son las que nos van a servir de base para el análisis bibliográfico de la producción científica de los profesionales de la Pedagogía Social desarrollado en este artículo.

\section{Producción científica del ámbito disciplinar}

\section{Seminario Interuniversitario de Pedagogía Social}

El formato que establece la Sociedad Iberoamericana de Pedagogía Social para el contacto entre los profesionales de su ámbito de conocimiento es el de Seminario.

Si consideramos un seminario como una reunión especializada, con naturaleza técnica y académica y cuyo objeto es realizar un estudio profundo de contenidos específicos y con un tratamiento que requiere una interactividad entre los especialistas, la denominación de Seminario Interuniversitario de Pedagogía Social, es muy acorde a los objetivos perseguidos. Así, los seminarios los podemos considerar como foros de docencia e investigación a la vez.

En la tabla 1, aparecen recogidas las cuatro etapas en las que, a juicio de Fermoso (2003a), pueden ser organizados los diferentes encuentros 
de Pedagogía Social. El citado autor discierne entre una primera etapa, comprendida entre 1981 y 1986; un segundo período, que abarca hasta 1993; un tercer momento, que alcanza hasta 2003 y, una última etapa, que incluye los encuentros acontecidos desde 2004 hasta nuestros días.

Tabla 1. Jornadas, Seminarios y Congresos de Pedagogía Social

\begin{tabular}{|c|c|c|c|c|c|}
\hline ETAPA & $\begin{array}{r}\text { SEMI- } \\
\text { NARIO }\end{array}$ & AÑO & LUGAR & TITULO & TEMA \\
\hline \multirow[t]{3}{*}{$\begin{array}{l}1^{\mathrm{a}}(1981- \\
1986)\end{array}$} & I & 1981 & Sevilla & $\begin{array}{l}\text { I Jornadas de P. S. } \\
\text { y Sociología de la } \\
\text { Educación }\end{array}$ & $\begin{array}{l}\text { Educación y } \\
\text { Sociedad }\end{array}$ \\
\hline & II & 1982 & $\begin{array}{l}\text { Santiago } \\
\text { de Com- } \\
\text { postela }\end{array}$ & $\begin{array}{l}\text { II Encuentro Interna- } \\
\text { cional de Sociología } \\
\text { de la Educación y P. S. }\end{array}$ & $\begin{array}{l}\text { Autonomías y } \\
\text { Educación }\end{array}$ \\
\hline & III & 1986 & Murcia & $\begin{array}{l}\text { III Jornadas Nacio- } \\
\text { nales de Pedagogía } \\
\text { Social. }\end{array}$ & $\begin{array}{l}\text { Se desconocen } \\
\text { las actas }\end{array}$ \\
\hline \multirow[t]{5}{*}{$\begin{array}{l}2^{a}(1987- \\
1993)\end{array}$} & IV & 1987 & $\begin{array}{l}\text { Santiago } \\
\text { de Com- } \\
\text { postela }\end{array}$ & $\begin{array}{l}\text { IV Jornadas de Peda- } \\
\text { gogía Social }\end{array}$ & $\begin{array}{l}\text { Diseño curricular- } \\
\text { conceptual y pro- } \\
\text { fesional en P. S. }\end{array}$ \\
\hline & V & 1988 & $\begin{array}{l}\text { Salaman- } \\
\text { ca }\end{array}$ & $\begin{array}{l}\text { Jornadas nacionales } \\
\text { de Pedagogía Social }\end{array}$ & $\begin{array}{l}\text { Educación Social } \\
\text { y Desarrollo }\end{array}$ \\
\hline & VI & 1990 & $\begin{array}{l}\text { Islas Ba- } \\
\text { leares }\end{array}$ & $\begin{array}{l}\text { Jornadas Nacionales } \\
\text { de Pedagogía Social }\end{array}$ & $\begin{array}{l}\text { Formación en la } \\
\text { empresa }\end{array}$ \\
\hline & VII & 1991 & Sevilla & $\begin{array}{l}\text { Jornadas Nacionales } \\
\text { de Pedagogía Social }\end{array}$ & $\begin{array}{l}\text { Se desconocen } \\
\text { las actas }\end{array}$ \\
\hline & VIII & 1992 & Murcia & $\begin{array}{l}\text { Jornadas Nacionales } \\
\text { de Pedagogía Social }\end{array}$ & $\begin{array}{l}\text { Se desconocen } \\
\text { las actas }\end{array}$ \\
\hline \multirow[t]{5}{*}{$\begin{array}{l}3^{\mathrm{a}}(1994- \\
2003)\end{array}$} & IX & 1994 & Valencia & $\begin{array}{l}\text { Seminario Interuni- } \\
\text { versitario de Pedago- } \\
\text { gía Social }\end{array}$ & $\begin{array}{l}\text { Se desconocen } \\
\text { las actas }\end{array}$ \\
\hline & $X$ & 1995 & Madrid & $\begin{array}{l}\text { Seminario Interuni- } \\
\text { versitario de Pedago- } \\
\text { gía Social }\end{array}$ & $\begin{array}{l}\text { El educador } \\
\text { social: profesión } \\
\text { y formación uni- } \\
\text { versitaria }\end{array}$ \\
\hline & XIX & 1996 & $\begin{array}{l}\text { Barcelo- } \\
\text { na }\end{array}$ & $\begin{array}{l}\text { Seminario Interuni- } \\
\text { versitario de Pedago- } \\
\text { gía Social }\end{array}$ & $\begin{array}{l}\text { Medios de } \\
\text { comunicación y } \\
\text { educación social }\end{array}$ \\
\hline & XII & 1997 & Deusto & $\begin{array}{l}\text { Seminario Interuni- } \\
\text { versitario de Pedago- } \\
\text { gía Social }\end{array}$ & $\begin{array}{l}\text { Nuevos espacios } \\
\text { de la Educación } \\
\text { Social }\end{array}$ \\
\hline & XIII & 1998 & Granada & $\begin{array}{l}\text { Seminario Interuni- } \\
\text { versitario de Pedago- } \\
\text { gía Social }\end{array}$ & $\begin{array}{l}\text { Políticas Socia- } \\
\text { les y Educación } \\
\text { Social }\end{array}$ \\
\hline
\end{tabular}


Contenidos y procesos en pedagogía social: deconstrucción de la última etapa de producción

José Antonio Rabadán Rubio y Encarnación Hernández Pérez

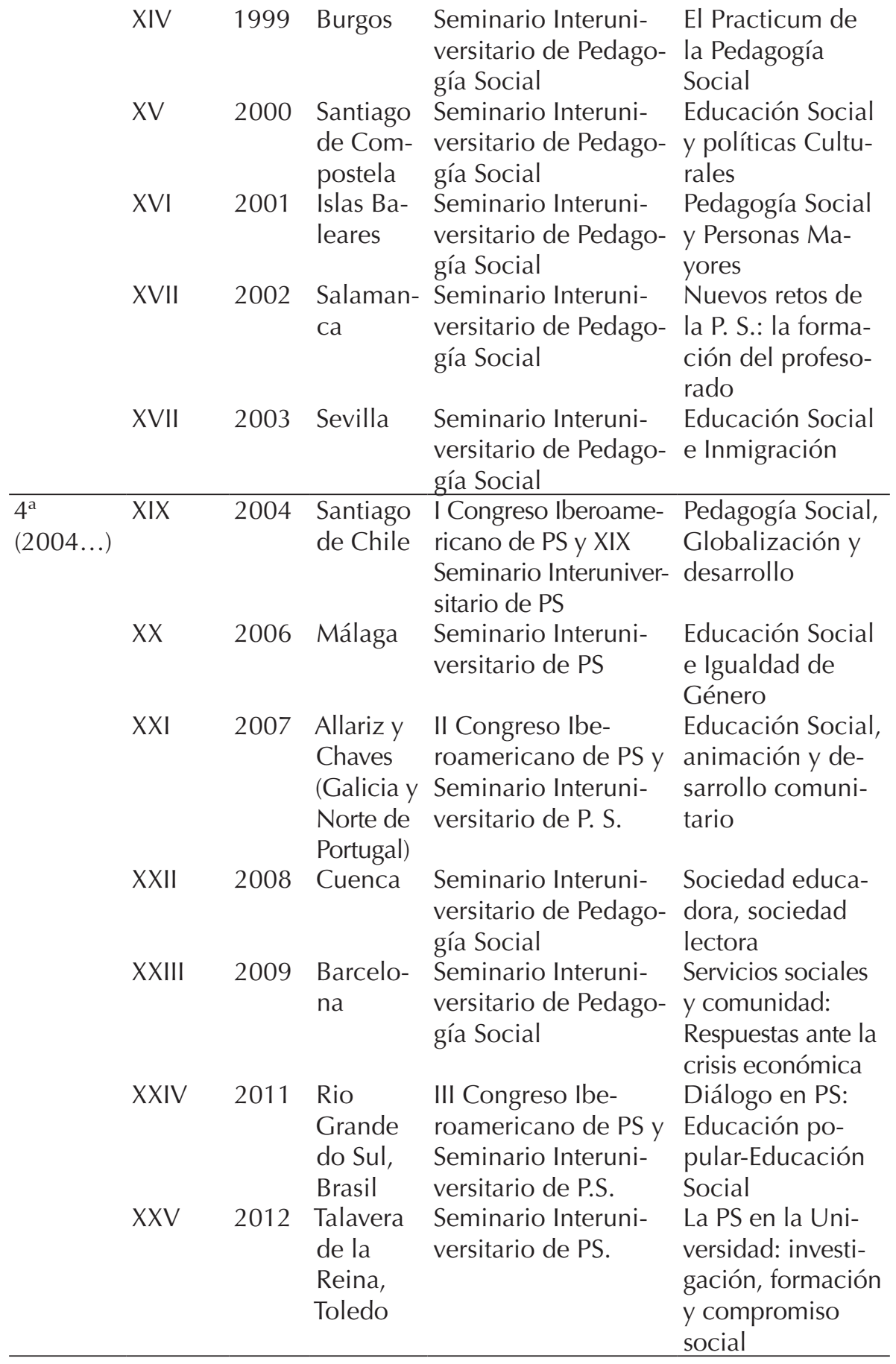


Tal y como figura en la tabla 1, todos los encuentros acontecidos bajo el paraguas de Pedagogía Social, han tenido una evolución y transformación en el tiempo. Siguiendo a Fermoso (2003a), podemos dividir los encuentros de Pedagogía Social en cuatro etapas. En una primera etapa (1981-1986) se celebraron tres Jornadas con temática dispar y con un punto de cohesión: la Sociología de la Educación; en la segunda (1987-1993), la producción científica es variada y escasa, así como la conservación de documentos y la denominación del evento pasa a ser Jornadas Nacionales de Pedagogía Social. En un tercer momento (19942003), este evento, aparece convocado como "Seminario Interuniversitario de Pedagogía Social", situación que ha continuado hasta el presente, existiendo una importante producción científica. Finalmente, podríamos considerar un cuarto período (2004 en adelante), al constituirse la Sociedad Ibérica de Pedagogía Social (fundada en el año 2000) como Sociedad Iberoamericana de Pedagogía Social y teniendo lugar la celebración del I Congreso Iberoamericano de Pedagogía Social en Santiago de Chile.

En el trabajo que nos ocupa, la última etapa es la que va a concentrar nuestros esfuerzos investigadores. Si hacemos coincidir la crisis económica y la burbuja inmobiliaria, en nuestros país en el año 2008, es esta fecha la utilizada para revisar temáticas y ámbitos abordados en los Seminarios de Pedagogía Social.

En la tabla 2, aparecen recogidas las comunicaciones presentadas en el congreso celebrado en 2007, bajo el título de "Educación social, animación y desarrollo comunitario", organizadas en función del eje temático y contenido de las mismas.

Tabla 2. II Congreso Iberoamericano de P.S. y Seminario Interuniversitario de P. S. (Allariz y Chaves)

\begin{tabular}{llll}
\hline \multicolumn{3}{c}{ EDUCACIÓN SOCIAL, ANIMACIÓN Y DESARROLLO COMUNITARIO } \\
\hline EJE TEMÁTICO & $\mathrm{N}^{\circ}$ COMUNICACIONES & \multicolumn{1}{c}{ CONTENIDOS } \\
\hline Animación & 24 & Epistemologicos & 12 \\
sociocultural, & & Epistemologico-Sociologico & 4 \\
educación y & Sociologico & 2 \\
globalización; & Sociologico- Pedagogico & 2 \\
& & Pedagogico & 2 \\
& & Epistemologico-Pedagogico & 2 \\
\hline
\end{tabular}


Contenidos y procesos en pedagogía social: deconstrucción de la última etapa de producción

José Antonio Rabadán Rubio y Encarnación Hernández Pérez

\begin{tabular}{llll}
\hline \multicolumn{1}{c}{ EJE TEMÁTICO } & $N^{\circ}$ COMUNICACIONES & \multicolumn{1}{c}{ CONTENIDOS } \\
\hline Educación y & 21 & Epistemologico & 3 \\
desarrollo comu- & & Epsitemologico-Sociologico & 7 \\
nitario rural y & Sociologico & 7 \\
urbano; & Sociologico-Pedagogico & 2 \\
& Pedagogico & 2 \\
\hline Animación y & 32 & Epistemologico & 17 \\
pedagogía del & & Epistemologico-Sociologico & 4 \\
tiempo libre; & Psicologico-Pedagogico & 3 \\
& Pedagogico & 4 \\
& Epistemologico-Pedagogico & 4 \\
\hline Profesionaliza- & 20 & Epistemologico & 11 \\
ción y experien- & Sociologico & 1 \\
cias de educa- & Epistemologico-Sociologico & 2 \\
ción, animación & Pedagogico & 5 \\
sociocultural y & Psicologico & 1 \\
desarrollo comu- & & \\
nitario. & & \\
\hline
\end{tabular}

El primero de los eventos que revisamos en este artículo, es el celebrado en el año 2007: "Educación social, animación y desarrollo comunitario", organizado por las Sociedades Iberoamericanas de Pedagogía Social, las universidades de Vigo, Tras-Os-Montes y Alto Douro,

En este encuentro, como podemos constatar en la tabla adjunta, coexisten cuatro ejes temáticos: Animación sociocultural, educación y globalización; Educación y desarrollo comunitario rural y urbano; Animación y pedagogía del tiempo libre y Profesionalización y experiencias de educación, animación sociocultural y desarrollo comunitario. Resulta especialmente significativa la prevalencia de los contenidos epistemológicos, frente al relativo protagonismo de las comunicaciones con un carácter pedagógico.

Un año más tarde, en 2008, Cuenca acoge el vigésimo segundo encuentro. Dado que nos hallamos ya en lo que Fermoso (2003a) cataloga como cuarta etapa, el acontecimiento adquiere la denominación de Seminario Interuniversitario de Pedagogía Social. Sociedad educadora, sociedad lectora, es la temática bajo la que se engloban las comunicaciones presentadas en el citado evento. En la tabla 3 se recoge información más detallada acerca de los ejes temáticos y contenidos en torno a los que versaron los trabajos presentados. 
Tabla 3. Seminario Interuniversitario de Pedagogía Social (Cuenca, 2008)

\begin{tabular}{|c|c|c|c|}
\hline \multicolumn{4}{|c|}{ SOCIEDAD EDUCADORA, SOCIEDAD LECTORA } \\
\hline EJE TEMÁTICO & No COMUNICACIONES & CONTENIDOS & \\
\hline $\begin{array}{l}\text { Ponencias } \\
\text { invitadas }\end{array}$ & 6 & Epistemologicos & 6 \\
\hline $\begin{array}{l}\text { Valores Socia- } \\
\text { les y lectura }\end{array}$ & 18 & $\begin{array}{l}\text { Epistemologicos } \\
\text { Epistemologicos-Pedagogicos } \\
\text { Epistemologicos-Sociologicos } \\
\text { Psicologicos } \\
\text { Pedagogicos }\end{array}$ & $\begin{array}{l}11 \\
1 \\
1 \\
1 \\
4\end{array}$ \\
\hline $\begin{array}{l}\text { La formación } \\
\text { de lectores }\end{array}$ & 10 & $\begin{array}{l}\text { Epistemologicos } \\
\text { Epistemologicos-Sociologicos } \\
\text { Sociologicos } \\
\text { Psicologicos } \\
\text { Epistemologicos-Psicologicos } \\
\text { Epistemologicos-Pedagogicos } \\
\text { Pedagogicos }\end{array}$ & $\begin{array}{l}2 \\
1 \\
1 \\
1 \\
1 \\
2 \\
2\end{array}$ \\
\hline $\begin{array}{l}\text { Lecturas y } \\
\text { medios de co- } \\
\text { municación }\end{array}$ & 8 & $\begin{array}{l}\text { Epistemologicos } \\
\text { Epistemologicos-Pedagogicos } \\
\text { Psicologicos } \\
\text { Pedagogicos }\end{array}$ & $\begin{array}{l}3 \\
1 \\
1 \\
3 \\
\end{array}$ \\
\hline $\begin{array}{l}\text { Animación } \\
\text { sociocultural, } \\
\text { educación y } \\
\text { globalización }\end{array}$ & 11 & $\begin{array}{l}\text { Epistemologicos } \\
\text { Epistemologicos-Sociologicos } \\
\text { Sociologicos } \\
\text { Pedagogicos }\end{array}$ & $\begin{array}{l}5 \\
2 \\
1 \\
3 \\
\end{array}$ \\
\hline $\begin{array}{l}\text { Educación } \\
\text { y desarrollo } \\
\text { comunitaria }\end{array}$ & 10 & $\begin{array}{l}\text { Epistemologicos } \\
\text { Epistemologico-Sociologico } \\
\text { Sociologico } \\
\text { Pedagogico } \\
\text { Epistemologico-Pedagogico }\end{array}$ & $\begin{array}{l}6 \\
1 \\
1 \\
1 \\
1 \\
\end{array}$ \\
\hline
\end{tabular}

De igual modo que en el encuentro anterior, se imponen las comunicaciones con un carácter epistemológico, hallándose nuevamente el contenido pedagógico poco presente en los trabajos presentados.

En 2009, es Barcelona la ciudad escogida para la celebración del XXIII encuentro, catalogado como Seminario Interuniversitario de Pedagogía Social. La temática escogida responde al título: Servicios Sociales y comunidad. Respuestas ante la crisis económica. En la tabla 4 aparecen recogidos los trabajos presentados para el citado acontecimiento. 
Contenidos y procesos en pedagogía social: deconstrucción de la última etapa de producción

José Antonio Rabadán Rubio y Encarnación Hernández Pérez

Tabla 4. Seminario interuniversitario de Pedagogía Social (Barcelona, 2009)

SERVICIOS SOCIALES Y COMUNIDAD:

RESPUESTAS ANTE LA CRISIS SOCIOECONOMICA

\begin{tabular}{|c|c|c|c|}
\hline EJE TEMÁTICO & $\begin{array}{l}\text { No COMUNI- } \\
\text { CACIONES }\end{array}$ & CONTENIDOS & \\
\hline $\begin{array}{l}\text { Servicios Sociales y aten- } \\
\text { ción socioeducativa en con- } \\
\text { textos de crisis económica }\end{array}$ & 14 & $\begin{array}{l}\text { Epistemológicos } \\
\text { Epistemologicos-Sociologicos } \\
\text { Sociologicos-Pedagógicos }\end{array}$ & \\
\hline $\begin{array}{l}\text { La acción comunitaria fren- } \\
\text { te a la crisis económica }\end{array}$ & 11 & $\begin{array}{l}\text { Epistemologicos } \\
\text { Epistemologicos-Sociologicos } \\
\text { Epistemologicos-Pedagogicos } \\
\text { Sociologicos } \\
\text { Pedagogicos }\end{array}$ & \\
\hline $\begin{array}{l}\text { Servicios Sociales, comuni- } \\
\text { dad y trabajo en red }\end{array}$ & 10 & $\begin{array}{l}\text { Epistemologicos } \\
\text { Epistemologicos-Sociologicos } \\
\text { Sociologicos-Pedaoggicos }\end{array}$ & \\
\hline
\end{tabular}

Una vez más, la epistemología se impone frente a contenidos pedagógicos, que, pese a lo que cabría esperar, dada la sociedad promotora del Seminario, apenas son abordados.

Finalmente, son recogidos en la tabla 5 los aspectos más relevantes del vigésimo cuarto encuentro, celebrado en Talavera de la Reina el pasado año.

Tabla 5. Seminario interuniversitario de Pedagogía Social (Toledo, 2012)

LA PS EN LA UNIVERSIDAD:

INVESTIGACIÓN, FORMACIÓN Y COMPROMISO SOCIAL

\begin{tabular}{llll}
\hline \multicolumn{1}{c}{ EJE TEMÁTICO } & $N^{\circ}$ COMUNICACIONES & \multicolumn{1}{c}{ CONTENIDOS } \\
\hline La Investigación & 26 & Epistemologico & 12 \\
en Pedagogía & & Sociologico & 10 \\
Social & & Sociologico-Pedagogico & 2 \\
& & Epistemologico-Pedagogico & 1 \\
& & Pedagogico & 1 \\
\hline La formación e & 22 & Epistemologico & 13 \\
investigación en & Sociologico & 3 \\
PS & Pedagogico & 5 \\
& Epistemologico-Pedagogico & 1 \\
\hline Impacto y com- & 25 & Epistemologico & 2 \\
promiso social de & Sociologico & 7 \\
la investigación & Pedagogico & 5 \\
en Pedagogía & Epistemologico-Pedagogico & 2 \\
Social & Pedagogico-Sociologico & 7 \\
& Sociologico-Psicologico & 2 \\
\hline
\end{tabular}


Bajo la temática La Pedagogía Social en la Universidad: Investigación, Formación y Compromiso Social, son recogidas un total de 73 comunicaciones, en las que si bien el carácter pedagógico de los trabajos parece haber ido aumentando, continua ocupando un discreto segundo plano.

\section{Pedagogía Social. Revista Interuniversitaria}

La Revista Interuniversitaria de Pedagogía Social, comienza sus andaduras en 1986 como una publicación plural y abierta a la reflexión y al debate sobre la Pedagogía Social y áreas afines, dando cuenta de los avances que se produzcan en la investigación sobre su objeto de estudio, la Educación Social, como indica la propia memoria de dicha revista en el año 2007. Se centra también en ámbitos afines de la acción e intervención socio-educativa. Abarca las dimensiones teórica, metodológica, teleológica y práctica.

La Revista Pedagogía Social. Revista Interuniversitaria se propone como tarea cumplir los siguientes objetivos:

a) Constituir un medio de expresión para la publicación de artículos relacionados con la Pedagogía Social y la Educación Social, así como de aquellas temáticas que les sean afines.

b) Promover la difusión de trabajos de investigación originales, auspiciar la construcción innovadora de temas y proyectos, así como la promoción de este campo de especialización entre la comunidad científica y académica.

c) Profundizar en el conocimiento de la realidad socioeducativa, principalmente en el contexto iberoamericano, a través de la difusión de trabajos de acreditada calidad científica.

d) Difundir artículos internacionales que marquen nuevas perspectivas para la investigación educativa en el ámbito de la Educación y la Pedagogía Social.

e) Fomentar la participación de los equipos y de los investigadores en esta área científica, propiciando la discusión sobre problemas relacionados con este campo de conocimiento teórico y empírico.

f) Contribuir al crecimiento del conocimiento asociado a la comprensión de la Educación Social, como objeto de estudio, como práctica y como profesión.

h) Difundir información de interés general o específico sobre resultados de investigaciones, libros y artículos publicados en otros medios. 
La Universidad de Murcia, bajo la Dirección del Dr. Juan Sáez Carreras, Catedrático de Pedagogía Social, fue la encargada de velar por el desarrollo de la misma desde sus inicios hasta el año 2006.

Son dos las épocas que ha pasado la revista desde sus inicios, en las que ha ido asentando sus bases para seguir avanzando en el presente y hacia el futuro que está por construir. En 2006 comenzaría la tercera época, en la que se encuentra en la actualidad.

En esta tercera época, se presta mayor atención a la realización de una autoevaluación, con el fin de tomar las medidas necesarias para lograr los índices de calidad e impacto que permitan un mayor reconocimiento de los trabajos que se publican.

Durante estos veintidós años, no ha dejado de publicarse. Se pretendía inicialmente la publicación de tres volúmenes anuales, pero la dificultad de mantener publicaciones de la realidad educativa e investigadora de este campo de calidad, las limitaciones que surgían y la necesidad de ajustar la Revista a los diferentes Criterios de Calidad del Proceso Editorial conllevaron a que se regulase el plazo de publicación de la Revista, siendo éste anual. En la tabla 6, se recogen las dos etapas en las que la producción de la revista Pedagogía Social, puede ser organizada, indicando el año de publicación y la temática de la misma.

Tabla 6. Publicaciones de la Revista Pedagogía Social (1986-2013)

\begin{tabular}{lllll}
\hline ETAPAS & $\begin{array}{l}\text { NOMBRE } \\
\text { REVISTA }\end{array}$ & AÑO & $\begin{array}{c}\text { NU- } \\
\text { MERO }\end{array}$ & \multicolumn{1}{c}{ TEMÁTICA } \\
\hline Primera & $\begin{array}{l}\text { Revista de } \\
\text { Etapa }\end{array}$ & 1986 & 1 & Epistemología Pedagogía Social \\
& Seciagogía & & 2 & El Menor \\
& 1988 & 3 & $\begin{array}{l}\text { Concepto, método y curriculum en } \\
\text { Pedagogía Social }\end{array}$ \\
& 1989 & 4 & Pedagogía Social en diferentes contextos \\
& 1990 & 5 & Educación para la paz \\
& 1991 & 6 & Educación y trabajo social \\
& 1992 & 7 & Pedagogía Social y desarrollo comuni- \\
& & & tario \\
& 1993 & 8 & Servicios sociales y educación \\
& 1994 & 9 & Educación de adultos \\
& 1995 & 10 & Educación cívica \\
& & 11 & Educación Social y Administraciones \\
& & & Públicas \\
& & 12 & Tercera edad \\
\hline
\end{tabular}




\begin{tabular}{|c|c|c|c|c|}
\hline ETAPAS & $\begin{array}{l}\text { NOMBRE } \\
\text { REVISTA }\end{array}$ & AÑO & $\begin{array}{c}\text { NU- } \\
\text { MERO }\end{array}$ & TEMÁTICA \\
\hline & & 1996 & 13 & Tercera edad II \\
\hline & & & 14 & Los derechos del niño \\
\hline & & 1997 & 15 & Pedagogía Social y mujer \\
\hline & & & 16 & Pedagogía Social y mujer \\
\hline \multirow{21}{*}{$\begin{array}{l}\text { Segun- } \\
\text { da etapa }\end{array}$} & \multirow{21}{*}{$\begin{array}{l}\text { Pedagogía } \\
\text { Social. } \\
\text { Revista } \\
\text { Interuni- } \\
\text { versitaria }\end{array}$} & 1998 & 1 & Drogas y Pedagogía Social \\
\hline & & & 2 & Educación ambiental \\
\hline & & 1999 & 3 & Interculturalidad \\
\hline & & & 4 & Interculturalidad \\
\hline & & 2000 & 5 & Los medios de comunicación \\
\hline & & & 6 & Exclusión Social \\
\hline & & 2001 & 7 & Exclusión Social \\
\hline & & & 8 & Políticas y Pedagogía Social \\
\hline & & 2002 & 9 & Pedagogía Social y voluntariado \\
\hline & & 2003 & 10 & Nueva conceptualización de la PS \\
\hline & & 2004 & 11 & $\begin{array}{l}\text { Homenaje a Constantino Mínguez. De } \\
\text { la Pedagogía Social a la Educación } \\
\text { Social }\end{array}$ \\
\hline & & 2005 & 12 & Educación para la salud \\
\hline & & 2006 & 13 & Educación para la salud \\
\hline & & 2007 & 14 & $\begin{array}{l}\text { La Pedagogía Social ante el proceso de } \\
\text { convergencia europea de la Educación } \\
\text { Superior }\end{array}$ \\
\hline & & 2008 & 15 & Educación social en la escuela \\
\hline & & 2009 & 16 & Competencias y profesionalización \\
\hline & & 2010 & 17 & $\begin{array}{l}\text { Representaciones sociales que influyen } \\
\text { en las políticas sociales de infancia y } \\
\text { adolescencia en Europa }\end{array}$ \\
\hline & & 2011 & 18 & Infocomunicación y Educación Social \\
\hline & & 2012 & 19 & Educación Social y Ética Profesional \\
\hline & & & 20 & Tiempos educativos y tiempos de ocio \\
\hline & & 2013 & 21 & Familia e intervención socioeducativa \\
\hline
\end{tabular}

De acuerdo con lo recogido en la tabla 6, en los 23 últimos años se han publicado un total de 37 números, con una temática muy variada, que abarcada desde monográficos dedicados a la infancia $(1986,1996)$ hasta otros que versan sobre la tercera edad (1995, 1996), o destinados a grupos susceptibles de exclusión $(2000,2001)$.

En la tabla 7 se recoge la producción de la revista Pedagogía Social en el último decenio, atendiendo al eje temático sobre el que versa y el 
Contenidos y procesos en pedagogía social: deconstrucción de la última etapa de producción

José Antonio Rabadán Rubio y Encarnación Hernández Pérez

tipo de contenido abordado (epistemológico, sociológico, pedagógico, psicológico).

Tabla 7. Producción de la Revista Pedagogía Social según tema y contenido

\begin{tabular}{|c|c|c|c|c|}
\hline \multicolumn{5}{|c|}{ Revista Interuniversitaria de Pedagogía Social } \\
\hline AÑO & EJE TEMÁTICO & $\begin{array}{c}\mathrm{N}^{\circ} \\
\text { ARTÍCULOS }\end{array}$ & CONTENIDOS & \\
\hline 2003 & $\begin{array}{l}\text { La pedagogía Social } \\
\text { a examen }\end{array}$ & 17 & $\begin{array}{l}\text { Epistemologicos } \\
\text { Epistemologicos-Sociologicos } \\
\text { Epistemologicos-pedagogicos } \\
\text { Sociologico } \\
\text { Pedagogico-Sociologico } \\
\text { Pedagogico }\end{array}$ & $\begin{array}{l}7 \\
1 \\
1 \\
2 \\
2 \\
4\end{array}$ \\
\hline 2004 & $\begin{array}{l}\text { De la Pedagogía So- } \\
\text { cial a la Educación } \\
\text { Social }\end{array}$ & 14 & $\begin{array}{l}\text { Epistemologico } \\
\text { Epistemologico-Sociologico } \\
\text { Sociologico } \\
\text { Sociologico-Pedagogico } \\
\text { Pedagogico } \\
\text { Psicologico-Pedagogico }\end{array}$ & $\begin{array}{l}2 \\
1 \\
5 \\
3 \\
2 \\
1\end{array}$ \\
\hline $\begin{array}{l}2005 / \\
2006\end{array}$ & $\begin{array}{l}\text { Educación para la } \\
\text { Salud }\end{array}$ & 18 & $\begin{array}{l}\text { Epistemologico } \\
\text { Epistemologico-Pedagogico } \\
\text { Sociologico } \\
\text { Sociologico-Pedagogico } \\
\text { Psicologico } \\
\text { Psicologico-Pedagogico } \\
\text { Pedagogico }\end{array}$ & $\begin{array}{l}6 \\
1 \\
5 \\
2 \\
1 \\
1 \\
2\end{array}$ \\
\hline 2007 & $\begin{array}{l}\text { Pedagogía Social } \\
\text { y convergencia } \\
\text { Europea }\end{array}$ & 10 & $\begin{array}{l}\text { Epistemologico } \\
\text { Epistemologico-Sociologico } \\
\text { Epistemologico-Pedagogico } \\
\text { Sociologico } \\
\text { Psicologico } \\
\text { Pedagogico }\end{array}$ & $\begin{array}{l}3 \\
2 \\
1 \\
1 \\
1 \\
2\end{array}$ \\
\hline 2008 & $\begin{array}{l}\text { Educación Social en } \\
\text { la escuela }\end{array}$ & 10 & $\begin{array}{l}\text { Sociologico } \\
\text { Sociologico-Psicologico } \\
\text { Psicologico } \\
\text { Pedagogico } \\
\end{array}$ & $\begin{array}{l}1 \\
1 \\
3 \\
5 \\
\end{array}$ \\
\hline 2009 & $\begin{array}{l}\text { Competencias y pro- } \\
\text { fesionalización }\end{array}$ & 10 & $\begin{array}{l}\text { Epistemologicos } \\
\text { Sociologicos } \\
\text { Sociologico-Pedagogico } \\
\text { Psicologico } \\
\text { Psicologico-Pedagogico }\end{array}$ & $\begin{array}{l}4 \\
1 \\
1 \\
3 \\
1\end{array}$ \\
\hline
\end{tabular}




\begin{tabular}{lllll}
\hline \multirow{2}{*}{ AÑO } & \multirow{2}{*}{ EJE TEMÁTICO } & \multicolumn{2}{c}{ No $^{\circ}$} & \multicolumn{1}{c}{ CONTENIDOS } \\
& ARTíCULOS & \\
\hline 2010 & Infancia y adoles- & 10 & Sociologico-Psicologico & 5 \\
& cencia en dificultad & & Sociologico-Pedagogico & 3 \\
& social & & Psicologico & 1 \\
& & & Psicologico-Pedagogico & 1 \\
\hline 2011 & Infocomunicación y & 10 & Sociologico-Pedagogico & 3 \\
& Educación Social & & Sociologico-Psicologico & 3 \\
& & Pedagogico & 1 \\
& & Psicologico-Pedagogico & 3 \\
\hline 2012 & Educación Social y & 10 & Epistemologicos & 6 \\
& Ética profesional & & Sociologico & 1 \\
& & Sociologico-Psicologico & 1 \\
& & Pedagogico & 1 \\
& & Pedagogico-Psicologico & 1 \\
\hline
\end{tabular}

Tal y como aparece reflejado en la tabla 7, un total de 109 artículos han sido publicados desde 2003. En los últimos años puede apreciarse el ligero incremento que los trabajos de carácter pedagógico (esencialmente o combinados con artículos con un tinte sociológico y/o psicológico) han experimentado. Así, de los artículos publicados en el período analizado, el 37\% (41 artículos) cuentan con un cariz pedagógico. Gran representación tienen, así mismo, los artículos con contenido epistemológico, representando el 32\% (35 artículos) de la producción de los 10 últimos años.

\section{Conclusiones}

La Pedagogía Social, y por ende, la figura del Educador Social, sigue siendo una figura profesional bastante desconocida e infravalorada, por la sociedad globalmente.

La educación social surge en el Estado de Bienestar y se desarrolla en el marco de unos estados que asumen la responsabilidad de satisfacer las necesidades básicas de la población (educación, salud, trabajo, vivienda, pensiones) fundamentalmente.

En la medida en que la globalización neoliberal tiende a la privatización de todos los servicios, incluidos los básicos, la Educación Social recibe el impacto de una serie de políticas que la colocan en la preca- 
Contenidos y procesos en pedagogía social: deconstrucción de la última etapa de producción

José Antonio Rabadán Rubio y Encarnación Hernández Pérez

riedad y el ostracismo político, administrativo y social, como nos indica Castillo (2004/5). Nuestro ámbito profesional lo podemos señalar como nuevo, aunque ya hoy, inmerso en una profunda crisis, cuestión esta que nos obliga a replantear la disciplina de forma distinta a la realizada hasta el momento. A la vez, a nivel de población general, presenta un alto grado de desconocimiento tanto para los ciudadanos como para otros profesionales, incluidos los propios de la comunidad educativa.

Así, podemos concluir que es preciso tomar conciencia de que no nos podemos quedar estancados, necesitamos ir cada día buscando nuevas vías y caminos en el proceso de sistematización del conocimiento y del ámbito de intervención educativa y, sobre todo, tras los importantes y drásticos cambios que está sufriendo nuestra sociedad, en estos cinco últimos años.

Ante la actual situación, y ante todas las que están apareciendo día a día, no podemos ni debemos mantenernos desidiosos, sino por el contrario, debemos plantearnos nuestras actuaciones donde la sociedad y los ciudadanos más nos necesitan, en los lugares donde las nuevas formas de exclusión y los conflictos están hoy más que nunca, presentes.

Por todo ello, hemos de incrementar nuestros niveles de reflexión y encauzar a nuestros alumnos a la búsqueda de los ámbitos laborales donde sean capaces de desarrollarse profesionalmente. Desde la Universidad, nos vemos en la obligación de ofertar a nuestros alumnos la formación necesaria científico-técnica para que puedan realizar un profundo análisis de la realidad, y así con ello, poder intervenir y transformarla desde una perspectiva educativa.

No podemos, ni debemos, dejar de lado nuestro "sustantivo", en el ámbito de la Pedagogía Social. El futuro es multidisciplinar y nuestra aportación, dentro de la multidisciplinaridad ha de ser, necesariamente, pedagógico.

Nuestra hipótesis de trabajo se inicia con el temor de que nuestro ámbito de conocimiento esté olvidando la "parte pedagógica" del corpus de conocimiento disciplinar y nos vamos quedando con líneas de investigación y profundización a nivel epistemológico, social y psicológico, dejando relegada a un gran ostracismo nada aconsejable la vertiente pedagógica, que ha de ser, en un futuro inmediato, la que signifique y profesionalice a nuestros alumnos, aportándoles un relevante significado, en la sociedad que hoy no nos identifica. 
Contenidos y procesos en pedagogía social: deconstrucción de la última etapa de producción

José Antonio Rabadán Rubio y Encarnación Hernández Pérez

El problema de nuestros tiempos es que el futuro ya no es lo que era. (Paul Valery, 1931)

\section{Referencias bibliográficas}

Augé, M. (1996). El sentido de los otros. Actualidad de la antropología. Barcelona, Paidós.

Becher, T. (2001). Tribus y territorios académicos: la indagación intelectual y las culturas de las disciplinas. Barcelona, Gedisa.

Bollnow, Otto F. (1969). Hombre y Espacio. Editorial Labor. S.A. Barcelona, España.

Castillo, A.J. (2004). Reflexiones en torno al futuro de la Educación Social y al papel del Educador Social en el contexto europeo. Revista Cuestiones Pedagógicas № 17 pp. 23-34.

Colom, A. (2003). ¿Una nueva epistemología en el devenir de la Educación Social? En Revista interuniversitaria de Pedagogía Social

Fermoso, P. (2003). Historia de la Pedagogía Social en España. Valencia: Nau Llibres

Fermoso, P. (2003). ¿Pedagogía Social o Ciencias de la Educación Social? En Pedagogía Social. Revista interuniversitaria. №10 Segunda Época. pp 61-84

Inkeles, A. (1968). ¿Qué es la sociología? Uteha, México, pp. 2-3

Arroyo, M. (1985). ¿Qué es la pedagogía social? Bordón, n²57. Madrid, pp. 203-215.

Ortega, J. (2005). Pedagogía Social y pedagogía escolar: la educación social en la escuela. Revista de Educación no336 (2005), pp. 111-127.

Perez Serrano, G. (2003). Pedagogía Social- Educación Social. Construcción científica e intervención práctica, Madrid, Narcea.

Rodriguez, A. (2006). Hacia una fundamentación epistemológica de la pedagogía social en Educación y Educadores, Vol 9, n² 2, pp.131-147

Torío-López, S. (2006). Evolución y desarrollo de la pedagogía social en España. Hacia una pedagogía social en construcción. Navarra. ESE 10, 37-54.

Sáez, J. (Coord.).(1993). El Educador Social. Universidad de Murcia, Servicio de Publicaciones

Sáez, J. (2003). La profesionalización de los Educadores Sociales (En busca de la competencia educativa cualificadora). Madrid. Dykinson, S.L.

Sáez, J. (2006). Pedagogía Social. Pensar la Educación Social como profesión. Madrid. Alianza.

Sáez, J. (2007). Pedagogía Social y Educación Social. Historia profesión y competencias. Pearson. Madrid. 
